\title{
Contributions of nursing in elderly care
}

\author{
Rosalina Aparecida Partezani Rodrigues
}

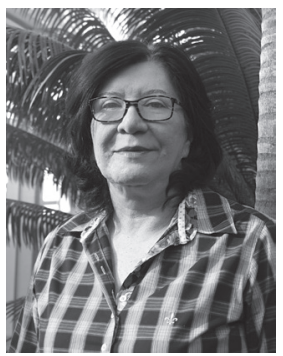

In the course of history, the population's social and health conditions have improved, but developing and underdeveloped countries still need to put in practice public policies to attend to the basic needs of human beings. Despite this condition, the reduced mortality and fecundity rates have served as important assessment indicators, increasing the life expectancy. In the global context, the longevity increase has doubled, but other situations have emerged that are associated with aging, such as chronic conditions and other geriatric syndromes. Against this background, Nursing faces a great challenge to act in the education process of appropriate human resources to deepen studies and research involving the elderly, in view of the population explosion.

Elderly care requires specific skills from Nursing, associated with an understanding of the biological, psychological, social and cultural theories that permeate aging, among others. The elderly are not "old people" with difficulties, but beings who have gone through important social experiences, with essential contributions to the development of society. Therefore, the Nurses' approach to elderly care should be implemented with respect, valuation of these human beings and understanding of their life process, beyond simple care for the disease or this aged body. Care is the essence of Nursing, but a new, emerging context is observed in health services, which is the elderly people's greater demand for care in this sector. Nevertheless, the question is raises: are the health services able to appropriately respond to the elderly people's health needs? How is Nursing organizing care at health services, including the family's participation in this network? Assessing elderly people's health conditions is a highly complex task as, besides the aspects of the senescence and senility process, the family arrangement and the care support network are influential, such as the available health services, the family and the elderly's income, the beliefs, values, among others.

Research development in Geriatric Nursing has advanced in Brazil, but difficulties remain for the development of multicenter research, due to the financial resources required for its development. In the analysis of research involving elderly people, the essence of the elderly as holistic beings should be considered, and not just their chronological age. The Brazilian culture and the understanding of the concept of elderly in the country should be taken into account, so that studies can be focused on the particularity of the country's context. The valuation of the researchers' role in this area is essential for human resource training, in teaching and research, as well as in high-quality scientific production, providing feedback to the care practice.

There is an urgent need for further research, mainly intervention studies, in the "locus of the elderly's life", keeping in mind that the family plays a preponderant role as these individuals' main caregivers; nevertheless, Nursing is responsible for presenting the evidence of this care and make it available to society. 
Research demonstrates that the families construct care typologies from a cultural perspective. Hence, the Nurses should get to know these typologies and propose elderly care models, based on a care framework in which the culture is included in this process ${ }^{(1)}$.

The Latin American Journal of Nursing takes interest in the dissemination of research results, mainly involving interventions, about elderly care, with a view to their immediate appropriation in practice.

\section{References}

1. Rodrigues RAP, Marques S, Kusumota L, Santos EB, Fhon JRS, Fabrício-Wehbe SCC. Transition of care for the elderly after cerebrovascular accidents - from hospital to the home. Rev. Latino-Am. Enfermagem. Jan.-Feb. 2013;21(spe):216-24.

Rosalina Aparecida Partezani Rodrigues is Associate Editor of the Revista Latino-Americana de Enfermagem and Full Professor of the Escola de Enfermagem de Ribeirão Preto, Universidade de São Paulo, WHO Collaborating Centre for Nursing Research Development, Ribeirão Preto, SP, Brazil, rosalina@eerp.usp.br 